\title{
Glucose Gel in Infants at Risk for Transitional Neonatal Hypoglycemia
}

\author{
Kartikeya Makker, MD ${ }^{1}$ Rana Alissa, MD ${ }^{1}$ Christopher Dudek, MD ${ }^{1}$ Laura Travers, DO ${ }^{1}$ \\ Carmen Smotherman, MS ${ }^{2}$ Mark L. Hudak, MD ${ }^{1}$
}

${ }^{1}$ Division of Neonatology, Department of Pediatrics, University of Florida College of Medicine-Jacksonville, Jacksonville, Florida

${ }^{2}$ Center for Health Equity and Research, University of Florida College of Medicine-Jacksonville, Jacksonville, Florida
Address for correspondence Kartikeya Makker, MD, Division of Neonatology, Department of Pediatrics, University of Florida College of Medicine-Jacksonville, 655 West 8th Street, Box C3, Jacksonville, FL 32209 (e-mail: kartikeya.makker@jax.ufl.edu).

Am J Perinatol 2018;35:1050-1056.

\begin{abstract}
Keywords

- glucose gel

- hypoglycemia

- breastfeeding

- transitional hypoglycemia

Objective To evaluate whether glucose gel as a supplement to feedings in infants admitted to the newborn nursery at risk for neonatal hypoglycemia $(\mathrm{NH})$ reduces the frequency of transfer to a higher level of care for intravenous dextrose treatment.

Study Design We revised our newborn nursery protocol for management of infants at risk for $\mathrm{NH}$ to include use of $40 \%$ glucose gel $(200 \mathrm{mg} / \mathrm{kg})$. Study population included late preterm, small and large for gestational age infants, and infants of diabetic mothers. We compared outcomes before $(4 / 1 / 14-3 / 31 / 15$ : Year 1$)$ and after $(4 / 1 / 15-$ 3/31/16: Year 2) initiation of the revised protocol. Our prospective primary outcome was transfer to the neonatal intensive care unit (NICU) for treatment with a continuous infusion of dextrose.

Results NICU transfer for management of NH fell from 8.1\% in Year 1 (34 of 421 at-risk infants screened) to $3.7 \%$ in Year 2 (14 of 383 at-risk infants screened). Rate of exclusive breastfeeding increased from $6 \%$ in Year 1 to $19 \%$ in Year 2. Hospital charges for the study population decreased from 801,276 USD to 387,688 USD in Year 1 and Year 2, respectively.

Conclusion Our study supports the adjunctive use of glucose gel to reduce NICU admissions and total hospitalization expense.
\end{abstract}

Achieving glucose homeostasis is one of the critical physiological sequences in the extrauterine adaptation of the neonate. Transient blood glucose concentrations as low as $30 \mathrm{mg} / \mathrm{dL}$ are common in the first 1 to 2 hours after birth, but generally rise to above $45 \mathrm{mg} / \mathrm{dL}$ by 12 hours of age. ${ }^{1-3}$ Neonatal hypoglycemia $(\mathrm{NH})$ occurs when blood glucose concentrations are substantially or persistently below these norms. Infants in the newborn nursery who are at increased risk for $\mathrm{NH}$ include late preterm, small for gestational age (SGA) and large for GA (LGA) infants, and infants of diabetic mothers (IDMs). $\mathrm{NH}$ is a common issue in the newborn nursery and the neonatal intensive care unit (NICU), affect-

received

August 5, 2017

accepted after revision

February 12, 2018

published online

March 26, 2018 ing 5 to $15 \%$ of otherwise healthy infants. ${ }^{4}$ Severe or prolonged $\mathrm{NH}$ can result in several systemic signs of illness and can increase the risk of long-term neurological injury. ${ }^{5,6}$ The Pediatric Endocrine Society and the American Academy of Pediatrics (AAP) disagree on the precise glucose concentration that defines $\mathrm{NH}$ at any point in time over the first few days after birth and/or that increases the risk of neurological injury. ${ }^{7,8}$

The AAP recommends treatment of certain blood glucose concentration with intravenous dextrose. ${ }^{7}$ Hence, a newborn with $\mathrm{NH}$ will undergo a potentially painful procedure, and in most institutions will require transfer to a higher level of care
Copyright $\odot 2018$ by Thieme Medical Publishers, Inc., 333 Seventh Avenue, New York, NY 10001, USA. Tel: +1(212) 584-4662.
DOI https://doi.org/ 10.1055/s-0038-1639338. ISSN 0735-1631. 
and consequent greater utilization of health care resources. Mother-infant separation, disrupted maternal-infant bonding, increased maternal anxiety, and breastfeeding failure are other undesired outcomes of treatment. For these reasons, other effective and less invasive options that might prevent the need to treat with intravenous dextrose are highly desirable.

Even though used in many resource-poor settings, 40\% glucose gel is not commonly used for prevention of $\mathrm{NH}$ in the developed world. ${ }^{9,10}$ In a benchmark study, buccal glucose was shown to decrease NICU admissions due to hypoglycemia, improve breastfeeding rates, and avoid breastfeeding failure associated with maternal infant separation. ${ }^{4}$ However, this study lacked ethnic diversity. ${ }^{11}$ More recently, a clinical quality improvement report documented the increased use of glucose gel in the United States. ${ }^{12}$ A recent Cochrane meta-analysis found no adverse effects of glucose gel during the neonatal period or at 2 years of age and recommended use of glucose gel as a first line approach for glycemic management of late preterm and term infants at risk for $\mathrm{NH}$ within the first 48 hours after birth. ${ }^{13}$ No randomized trial of glucose gel has been reported in the United States in populations at risk for NH. Our retrospective study of the use of oral glucose gel for prevention of $\mathrm{NH}$ is the largest study so far conducted in the United States.

\section{Materials and Methods}

We conducted a retrospective study to compare outcomes in infants at risk for $\mathrm{NH}$ admitted to the newborn nursery for the 1-year period before (Year 1) and the 1-year period after (Year 2) introduction on April 1, 2015 of a protocol that prescribed oral glucose gel as an adjunctive therapy. Our institutional pharmacy secured $40 \%$ glucose gel in prepackaged tubes of $15 \mathrm{~g}$ at a cost of 4.6 USD per tube. The primary study outcome was the rate of transfer of infants in the study population to the NICU for treatment of NH with a continuous infusion of D10W. Secondary study outcomes included the rate of exclusive breastfeeding (EBF; defined by World Health Organization as no other food or drink, not even water, except breast milk, including maternal expressed milk or milk from a wet nurse) for 6 months of life, but allows the infant to receive oral rehydration solution drops and syrups (vitamins, minerals, and medicines) ${ }^{14}$ and total NICU charges in the at-risk study population.

Our pregel institutional protocol for management of newborn nursery infants at risk for $\mathrm{NH}$ was in accord with extant AAP recommendations. ${ }^{7}$ We monitored LGA and IDM infants for a minimum of 12 hours and late preterm and SGA infants for a minimum of 24 hours. We discontinued monitoring if an infant had acceptable glucose concentration for $\geq 2$ consecutive measurements. Point-of-care glucose measurement was performed using the Accu-Check Inform II system (model no. 04882458001, Roche Diagnostics USA) glucometer.

Our management protocols specified interventions contingent upon measured glucose concentration. During Year 1, infants less than 4 hours of age were treated with a $2 \mathrm{~mL} / \mathrm{kg}$ bolus of intravenous D10W for a glucose concentration $<25 \mathrm{mg} / \mathrm{dL}$ after the initial feed. Early or additional feeds were offered if glucose concentration was 25 to $39 \mathrm{mg} / \mathrm{dL}$. After the first 4 hours of life, infants with glucose concentration $<35 \mathrm{mg} / \mathrm{dL}$ received a $2 \mathrm{~mL} / \mathrm{kg}$ bolus of intravenous D10W. Early or additional feeds were offered if glucose concentration was 35 to $44 \mathrm{mg} / \mathrm{dL}$ (-Fig. 1). If an infant met criteria to receive a second bolus of D10W, the infant was transferred to the NICU for further management

In Year 2, our revised protocol incorporated adjunctive administration of glucose gel $(200 \mathrm{mg} / \mathrm{kg}$ or $0.5 \mathrm{~mL} / \mathrm{kg}$ ) immediately before feeding at every intervention point in our former protocol that had called for an early or additional feeding (-Fig. 1). As in the Year 1 protocol, an infant received an intravenous bolus of $2 \mathrm{~mL} / \mathrm{kg}$ D10W if the criteria were met. We allowed at most four gel treatments and one bolus of D10W in the nursery. If a second bolus of D10W was indicated, it was given in the nursery and the baby was immediately transferred to the NICU for treatment with a continuous intravenous infusion of D10W.

A team of physicians conducted educational sessions for the nursing staff that highlighted the potential benefits of gel treatment and the proper technique of buccal administration. We then used the "train the trainer" model over a 2month period before the gel was formally introduced into practice on April 1, 2015.

We included infants in this study who were $\geq 35$ weeks' $\mathrm{GA}$ and $\geq 2,000 \mathrm{~g}$ birth weight and at increased risk for hypoglycemia (LGA, SGA, late preterm [GA, 350/7-36 6 6/7 weeks], and/or IDM). Exclusion criteria included transfer to the NICU for an indication other than hypoglycemia (e.g., sepsis, respiratory distress at birth), suspicion of a genetic disorder, and failure to adhere to our glucose management protocol. The Institutional Review Board at the University of Florida College of Medicine (UFCOM) - Jacksonville approved this retrospective study.

Statistical support was provided by the Center for Health Equity and Quality Research (CHEQR) at UFCOM - Jacksonville. Descriptive summaries were frequencies and percentages for categorical variables, and means, standard deviations, or medians and quartiles for continuous variables. Comparisons of demographics and study outcomes between study population infants in Year 1 and Year 2 were made using the Pearson's chi-square test (or Fisher's exact test, if appropriate) for categorical data, and the Wilcoxon rank sum test for the continuous data. The level of significance was set at 0.05. All analyses were performed using SAS for Windows Version 9 (SAS Version 9.4 for Windows, SAS Institute Inc., 2008, Cary, NC).

\section{Results}

\section{General Results}

The study population comprised 804 infants among whom 421 (52\%) were admitted to the newborn nursery in Year 1 and 383 (48\%) in Year 2. Demographic characteristics of infants were similar in the two 1-year periods (-Table 1) except for higher number of late preterm infants in Year 1. 


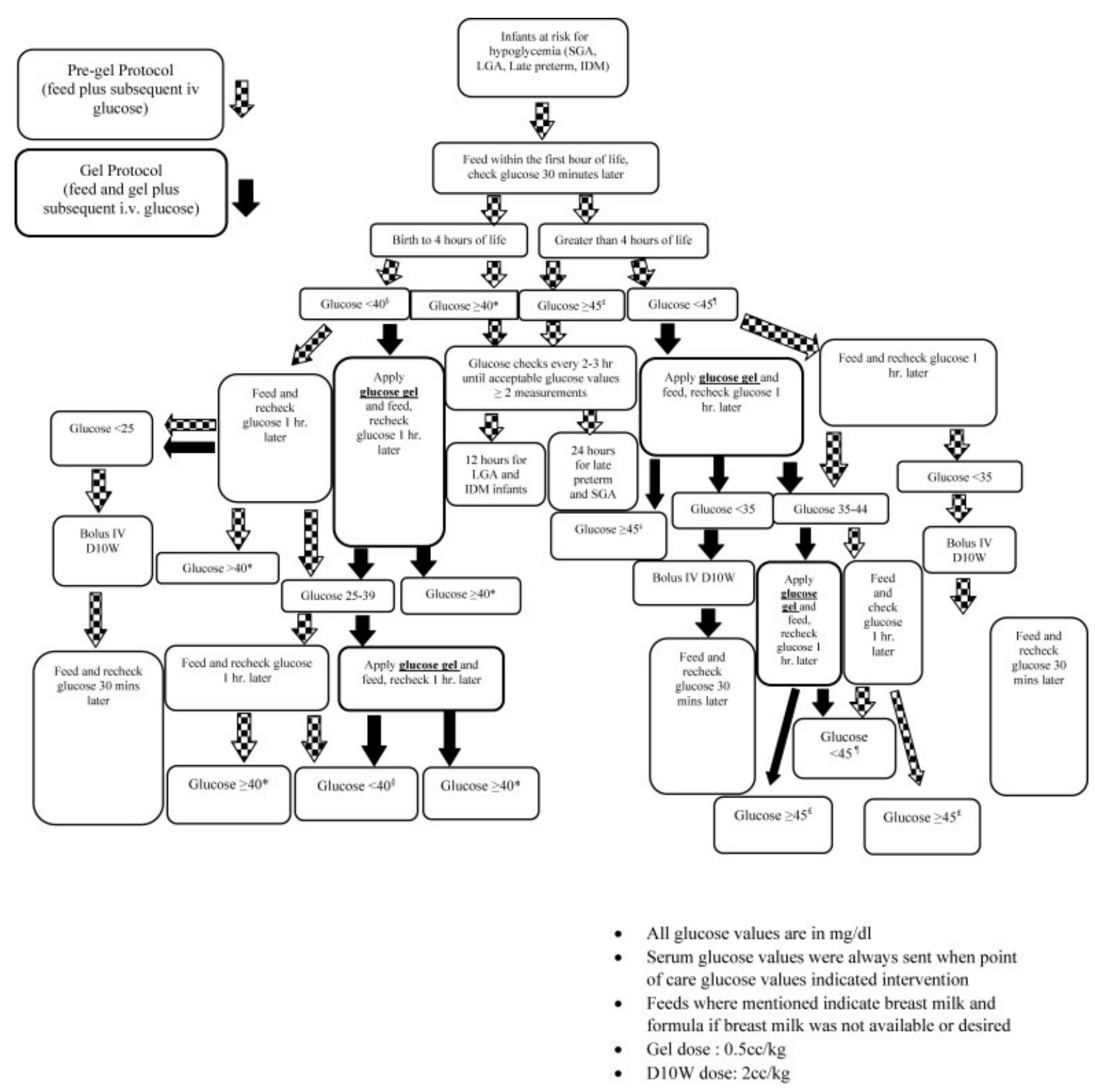

Fig. 1 University of Florida College of Medicine-Jacksonville Newborn Nursery Hypoglycemia Management Protocol.

However, in the subset of infants admitted to NICU, the rate of late preterm infants did not differ between Year $1(n=13$, $38 \%)$ and Year $2(n=7,50 \%, p=0.45)$. The distribution of risk factors for $\mathrm{NH}$ also did not differ during the two periods. In Year 1, we documented 253 episodes of hypoglycemia (glucose concentration $\leq 45 \mathrm{mg} / \mathrm{dL}$ ) with 30 episodes with glucose concentration $\leq 25 \mathrm{mg} / \mathrm{dL}$. In Year 2, there were 305 episodes of hypoglycemia of which 37 episodes had glucose concentration $\leq 25 \mathrm{mg} / \mathrm{dL}$. The incidence of glucose concentration $\leq 25 \mathrm{mg} / \mathrm{dL}$ did not differ in Year 2 compared with Year 1 , whether indexed to the total number of hypoglycemic events or to the total number of infants at risk for hypoglycemia. In Year 2, of the infants who received a glucose gel treatment and remained in the nursery, the last glucose measurement occurred at a mean age of 24 hours of life (range,
11-38 hours). Because these infants had a median length of stay (LOS) of 2 days in the newborn nursery before discharge home (-Table 2), we observed them on average $\sim 24$ hours beyond completion of the hypoglycemia protocol.

\section{Effect on NICU Admissions}

Introduction of glucose gel as an adjunctive therapy reduced the percentage of study population babies who required transfer to the NICU for continuous intravenous infusion of D10W from $8.1 \%$ in Year 1 to $3.7 \%$ in Year $2(p=0.01$, - Table 2$)$ and reduced the odds ratio (OR) of transfer to the NICU by $57 \%$ (OR $=0.43,95 \%$ confidence interval $[\mathrm{CI}]: 0.22,0.83)$. Of the 161 infants who received glucose gel, 73 infants (45\%) received 1 gel, 48 infants (30\%) received 2 gels, 28 infants (17\%) received 3 gels, and 12 infants (8\%) received 4 gel doses. Of 
Table 1 Demographics

\begin{tabular}{|c|c|c|c|}
\hline & $\begin{array}{l}\text { Year } 1 \\
(n=421,52 \%)\end{array}$ & $\begin{array}{l}\text { Year } 2 \\
(n=383,48 \%)\end{array}$ & $p$-Value \\
\hline Birth weight $(g)^{a}$ & $2,984(670)$ & $2,996(657)$ & $0.76^{c}$ \\
\hline$G A(w k)^{a}$ & $37.4(1.8)$ & $37.7(1.7)$ & $0.08^{c}$ \\
\hline Female gender & $197(47)$ & $199(52)$ & $0.14^{\mathrm{d}}$ \\
\hline African American & $232(55)$ & $219(57)$ & $0.70^{\mathrm{d}}$ \\
\hline Non-Hispanic & $362(86)$ & $337(88)$ & $0.62^{d}$ \\
\hline Vaginal delivery & $268(64)$ & $240(63)$ & $0.77^{d}$ \\
\hline $5 \mathrm{~min} \mathrm{APGAR}^{\mathrm{b}}$ & $9(9,9)$ & $9(9,9)$ & $0.32^{c}$ \\
\hline Infant of diabetic mother & $108(26)$ & $120(31)$ & $0.07^{d}$ \\
\hline Large for gestational age & $45(11)$ & $43(11)$ & $0.82^{d}$ \\
\hline Small for gestational age & $140(33)$ & $128(33)$ & $0.96^{d}$ \\
\hline Late preterm infant & $168(40)$ & $126(33)$ & $0.03^{\mathrm{d}, \mathrm{e}}$ \\
\hline
\end{tabular}

Abbreviations: APGAR, Appearance, Pulse, Grimace, Activity, and Respiration; GA, gestational age.

Note: Data are presented as counts (percentages), unless otherwise specified.

a Mean (SD).

${ }^{\mathrm{b}}$ Median (1st quartile, 3rd quartile).

'Wilcoxon rank sum test.

'Pearson's chi-square.

$\mathrm{e}_{p}<0.05$.

these, 14 were transferred to the NICU. The infants transferred to the NICU did not differ from the other 147 infants treated with gel who remained in the newborn nursery with the exception of glucose concentration (-Table 3 ). The mean glucose concentration in infants who failed the gel therapy was lower than in infants who responded favorably (30.4 vs. $34.8 \mathrm{mg} / \mathrm{dL} ; p=0.002$ ). The number of glucose gel treatments did not correlate with the likelihood of NICU admission $(p=0.78,-$ Table 3$)$.

\section{Gel Use and EBF}

Among the study population, the rate of EBF increased significantly from $6 \%$ in Year 1 to $19 \%$ in Year 2
( $p<0.001$, - Table 2). However, there was no statistical difference in rates of NICU admission in Year 1 between EBF infants and non-EBF infants. Similarly, for Year 2 there was no statistical difference in NICU admissions and gel use in EBF and non-EBF infants. We did not assess EBF rates after discharge.

\section{Effect on Health Care Charges}

In the state of Florida, most payers including Medicaid bundle normal newborn charges into the mother's hospital charges. Hospital charges for NICU care are billed separately. The hospital charges for this study population decreased from 801,276 USD in Year 1 to 387,688 USD in Year 2

Table 2 Clinical outcomes

\begin{tabular}{|c|c|c|c|}
\hline & $\begin{array}{l}\text { Year } 1 \\
(n=421,52 \%)\end{array}$ & $\begin{array}{l}\text { Year } 2 \\
(n=383,48 \%)\end{array}$ & $p$-Value \\
\hline NICU admission & $34(8)$ & $14(4)$ & $0.01^{\mathrm{b}, \mathrm{c}}$ \\
\hline Exclusive breastfeeding rates & $27(6)$ & $73(19)$ & $<0.001^{\mathrm{b}, \mathrm{c}}$ \\
\hline NICU length of stay (LOS) in days ${ }^{a}$ & $5.5(3,11)$ & $4(3,10)$ & $0.45^{d}$ \\
\hline Nursery LOS for babies admitted eventually to $\mathrm{NICU}^{\mathrm{a}}$ & $1(0,2)$ & $1(1,1)$ & $0.95^{d}$ \\
\hline Nursery LOS for babies not admitted to NICU & $2(2,2)$ & $2(2,2)$ & $0.13^{b}$ \\
\hline Infants receiving any D10 bolus & $25(6)$ & $24(6)$ & $0.84^{\mathrm{b}}$ \\
\hline Infants admitted to NICU after receiving D10 bolus & $17(68)$ & $9(38)$ & $<0.001^{\mathrm{b}, \mathrm{c}}$ \\
\hline
\end{tabular}

Abbreviation: NICU, neonatal intensive care unit.

Note: Data are presented as counts (percentages) unless specified.

aMedian (1st quartile, 3rd quartile).

bPearson's chi-square.

${ }^{\mathrm{c}} p<0.05$.

dWilcoxon rank sum test. 
Table 3 Comparison of characteristics of infants who received at least one treatment of glucose gel

\begin{tabular}{|l|l|l|l|}
\hline & $\begin{array}{l}\text { NICU admissions } \\
(\boldsymbol{n}=\mathbf{1 4}, \mathbf{9 \%})\end{array}$ & $\begin{array}{l}\text { No NICU admissions } \\
(\boldsymbol{n}=\mathbf{1 4 7 , 9 1 \% )}\end{array}$ & $p$-Value \\
\hline Birth weight $(\mathrm{g})^{\mathrm{a}}$ & $3,151(643)$ & $3,084(703)$ & $0.58^{\mathrm{c}}$ \\
\hline GA (wk) & $36.8(1.8)$ & $37.5(1.6)$ & $0.10^{\mathrm{c}}$ \\
\hline Female gender & $9(64)$ & $74(50)$ & $0.41^{\mathrm{d}}$ \\
\hline African American & $5(36)$ & $71(48)$ & $0.43^{\mathrm{d}}$ \\
\hline Non-Hispanic & $13(93)$ & $131(89)$ & $1.00^{\mathrm{d}}$ \\
\hline Vaginal delivery & $9(64)$ & $89(61)$ & $1.00^{\mathrm{d}}$ \\
\hline 5 min APGAR & $8.9(0.3)$ & $8.8(0.6)$ & $0.64^{\mathrm{c}}$ \\
\hline Infant of diabetic mother & $5(36)$ & $54(37)$ & $1.00^{\mathrm{d}}$ \\
\hline Large for gestational age & $3(21)$ & $20(14)$ & $0.43^{\mathrm{d}}$ \\
\hline Small for gestational age & $3(21)$ & $39(27)$ & $1.00^{\mathrm{d}}$ \\
\hline Late preterm & $7(50)$ & $51(35)$ & $0.383^{\mathrm{e}}$ \\
\hline Glucose value for gel intervention & \\
\hline Number of gels: 1 & $30.4(5.0)$ & $34.8(7.6)$ & $0.002^{\mathrm{c}, \mathrm{e}}$ \\
\hline Number of gels: 2 & 6 & 62 & $0.78^{\mathrm{d}}$ \\
\hline Number of gels: $\geq 3$ & 3 & 43 & \\
\hline
\end{tabular}

Abbreviations: APGAR, Appearance, Pulse, Grimace, Activity, and Respiration; GA, gestational age; NICU, neonatal intensive care unit. Note: Data are presented as counts (percentages), unless otherwise specified.

${ }^{\mathrm{a}}$ Mean (SD).

bMedian (1st quartile, 3rd quartile).

'Wilcoxon rank sum test.

'Fisher's exact test.

$\mathrm{e}_{p}<0.05$.

(-Table 4). Professional charges for only the NICU admissions decreased from 117,835 USD in Year 1 to 59,020 USD in Year 2.

\section{Other Secondary Outcomes}

LOS in the NICU for infants transferred for hypoglycemia management in Year 1 (median, 5.5 days, quartiles: 3 days, 11 days) did not differ from that of infants in Year 2 (median, 4 days, quartiles: 3 days, 10 days) (-Table 2). For infants transferred to the NICU, LOS in the nursery prior to admission during both periods was not different. The LOS in the nursery for at-risk infants not admitted to the NICU was also similar with a median stay of 2 days during both 1 -year periods (-Table 2). The number of infants receiving an intravenous bolus of D10W did not differ statistically during the two periods ( $p=0.84$ ). However, $68 \%$ of those receiving a bolus during Year 1 were admitted to the NICU versus 38\% in Year 2 $(p<0.001)$.

\section{Discussion}

Significant NH can cause brain injury that later manifests with neurodevelopmental deficits, but the effect of milder $\mathrm{NH}$ on long-term neurodevelopmental outcomes is not well defined. Although identification and timely management of recurrent or persistent nontransitional hypoglycemia during infancy improves long-term outcomes, ${ }^{15}$ there is no evidence that treatment of infants with asymptomatic $\mathrm{NH}$ has the same effect. ${ }^{16}$ Currently, $30 \%$ of all infants have risk factors for transitional $\mathrm{NH}$ and undergo glucose screening after birth. About $10 \%$ of screened infants in the United States require higher level care for glycemic management ${ }^{4,17}$ at an

Table 4 Economic impact of gel use

\begin{tabular}{|l|l|l|}
\hline & Year 1 & Year 2 \\
\hline Total professional charges for infants admitted to NICU & 59,020 \\
\hline Professional charges per 1,000 nursery admissions ${ }^{\text {a }}$ & 117,835 & 21,642 \\
\hline Total hospital charges for infants admitted to NICU & 42,601 & 387,688 \\
\hline Hospital charges per 1,000 nursery admissions ${ }^{\text {a }}$ & 801,276 & 143,216 \\
\hline
\end{tabular}

Abbreviation: NICU, neonatal intensive care unit.

${ }^{a}$ All charges are in US dollars. 
annual cost of 2.1 billion USD. ${ }^{18}$ Disagreements persist about the glucose concentration at which treatment might be indicated. In children and adults, a blood glucose concentration below $60 \mathrm{mg} / \mathrm{dL}$ is considered to be abnormal. ${ }^{19}$ The Children with Hypoglycemia and their Later Development (CHYLD) study reported no adverse neurologic outcomes at 2 years of age when the glucose concentration that prompted intervention was no less than $47 \mathrm{mg} / \mathrm{dL} .{ }^{20} \mathrm{~A}$ follow-up report of the Sugar Babies Study also provided evidence that use of glucose gel was not associated with adverse outcomes at 2 years corrected age. ${ }^{21}$ The Canadian Pediatric Society guidelines $^{22}$ prescribe intervention for single glucose concentration $<1.8 \mathrm{mmol} / \mathrm{L}(<32 \mathrm{mg} / \mathrm{dL})$ and repeated $(\geq 2)$ concentration $<2.6 \mathrm{mmol} / \mathrm{L}(<47 \mathrm{mg} / \mathrm{dL})$.

AAP guidelines call for an initial approach of early and/or frequent feeds (with human milk or formula) with treatment escalation to bolus or continuous infusion of intravenous dextrose if glucose concentrations are significantly or persistently low. ${ }^{7}$ Establishing intravenous access may cause pain and stress to a neonate, increase health care workload and costs, and impair maternal-infant bonding and establishment of breastfeeding. ${ }^{4} \mathrm{~A}$ recent report describes that introduction of glucose gel as an adjunctive therapy decreased the frequency of transfer to the NICU for management of NH among other benefits. ${ }^{23}$ We report the largest U.S. experience to date with glucose gel as an adjunctive therapy for infants at risk for $\mathrm{NH}$.

In our study, the percentage of infants at risk for transitional NH transferred to the NICU for management of hypoglycemia fell by more than $50 \%$ from Year 1 to Year 2 . While the percentage of the study population who received a bolus of D10W bolus did not differ between the 2 years, the rate of NICU transfer for infants who received a D10W bolus during Year 2 was lower than during Year 1 ( - Table 2). This suggests that glucose gel was instrumental in achieving extended normoglycemia after the acute effects of the D10W bolus abated. Not surprisingly, the mean glucose concentration of infants who required transfer to the NICU was lower than that in infants who maintained acceptable concentration after gel treatment.

The LOS of neonates who remained in the nursery for the full duration of hospitalization after receiving any glucose gel was comparable to the LOS of infants managed with feeds alone, so that the greater monitoring and higher complexity of care associated with use of glucose gel did not prolong hospital stay. In addition, we found no evidence that use of the gel prolonged stay in the newborn nursery before transfer to the NICU when indicated.

In our innercity hospital, EBF rates are lower than that reported in Florida and U.S. national rates. ${ }^{24}$ EBF rates for infants at risk for $\mathrm{NH}$ increased threefold after the introduction of the adjunctive glucose gel therapy. From Year 1 to Year 2 , our overall newborn nursery EBF rate increased from 21 to $35 \%$ (not reported in table). This rise is more than could be accounted for by greater breastfeeding success in the study population. Many other factors, including increased focus on breastfeeding promotion by new nursing leaders, introduction of donor breast milk as an option for mothers committed to exclusive nursing, and increased support from certified lactation consultants, were more influential in achieving this success than the use of glucose gel. Efforts are ongoing to continue to improve our institutional EBF rate.

Our economic impact data quantitate professional and hospital charges for the study population billed during the course of care in both the newborn nursery and the NICU. We found a $50 \%$ reduction in both sets of charges. Additionally, the low cost of gel further rationalizes its use as a first line intervention for $\mathrm{NH}$.

We acknowledge that the nonrandomized uncontrolled and sequential nature of our study are the limitations. In this setting, unquantified changes in clinical management attendant to the introduction of a new protocol may have improved care in other ways and magnified the effect of the structured intervention. Also, this was a short-term inpatient study and was not designed to evaluate longterm developmental outcomes.

Other real-world factors may impede the ability of other centers to replicate the magnitude of our treatment effect. First, we allowed for administration of a maximum of four gel treatments, which is higher than the number reported in a previous study ${ }^{23}$ but lower than the maximum of six gels allowed in the Sugar Baby Trial ${ }^{4}$ (treatment failure was defined as blood glucose concentration of less than 2.6 $\mathrm{mmol} / \mathrm{L} 30$ minutes after the second of the 2 doses of gel). The decision to use four gels in our study was based on input from nursing administration about nursing workload and the fact that our protocol does accommodate a D10W bolus in our nursery. Second, we believe that it is uncommon that other hospitals continue to monitor infants in the newborn nursery after a single intravenous bolus of D10W. The use of D10W has been part of our newborn nursery hypoglycemia management protocol before the introduction of glucose gel. We do recommend that units planning to implement use of glucose gel should carefully set the maximum number of gel treatments and D10W use in consideration of local nursing resources and anticipated workload to achieve operational success. Third, our hypoglycemia protocol and study design limited our ability to determine the change in glucose concentration in response to the gel administration. Use of intravenous D10W bolus prior to gel use in some infants confounds the assessment of glucose response after a subsequent use of glucose gel. However, a recent study has confirmed that the glucose concentration increases significantly after a gel application (mean increase by $11.7 \mathrm{mg} / \mathrm{dL}$ [95\% CI: 10.4-12.8]) and that breastfeeding is associated with reduced requirement of repeat gel treatment. ${ }^{25}$ This randomized trial also highlighted that glucose concentration increased by a greater amount after formula than after breast milk (expressed or nursed) or no milk $(p=0.004) .{ }^{25}$ Fourth, the retrospective nature of our study and nonuniform choice of formula allowed under the protocol prevented an assessment of a differential response to glucose gel in breast milk versus formula fed infants.

Accurate quantitation of the effect of adjunctive glucose gel therapy awaits completion of a planned randomized U.S. trial (NCT02523222). ${ }^{20}$ This and other future trials should 
provide more robust information on the optimal dose and timing of glucose gel therapy and on longer term outcomes that will further refine future implementation.

\section{Conflict of Interest}

None.

\section{Acknowledgments}

We thank Mr. David Tomlin, Application Analyst, for helping extract list of at-risk infants from the electronic medical record which helped us with data extraction. We also acknowledge Ms. Vanessa Martin, Division Manager, UFCOM Neonatology, for assistance in editing and submission of the article.

\section{References}

1 Srinivasan G, Pildes RS, Cattamanchi G, Voora S, Lilien LD. Plasma glucose values in normal neonates: a new look. J Pediatr 1986;109 (01):114-117

2 Heck LJ, Erenberg A. Serum glucose levels in term neonates during the first 48 hours of life. J Pediatr 1987;110(01):119-122

3 Hoseth E, Joergensen A, Ebbesen F, Moeller M. Blood glucose levels in a population of healthy, breast fed, term infants of appropriate size for gestational age. Arch Dis Child Fetal Neonatal Ed 2000;83 (02):F117-F119

4 Harris DL, Weston PJ, Signal M, Chase JG, Harding JE. Dextrose gel for neonatal hypoglycaemia (the Sugar Babies Study): a randomised, double-blind, placebo-controlled trial. Lancet 2013;382 (9910):2077-2083

5 Hay WW Jr, Raju TNK, Higgins RD, Kalhan SC, Devaskar SU. Knowledge gaps and research needs for understanding and treating neonatal hypoglycemia: workshop report from Eunice Kennedy Shriver National Institute of Child Health and Human Development. J Pediatr 2009;155(05):612-617

6 Cornblath M, Hawdon JM, Williams AF, et al. Controversies regarding definition of neonatal hypoglycemia: suggested operational thresholds. Pediatrics 2000;105(05):1141-1145

7 Adamkin DH; Committee on Fetus and Newborn. Postnatal glucose homeostasis in late-preterm and term infants. Pediatrics 2011;127(03):575-579

8 Thornton PS, Stanley CA, De Leon DD, et al; Pediatric Endocrine Society. Recommendations from the Pediatric Endocrine Society for Evaluation and Management of Persistent Hypoglycemia in Neonates, Infants, and Children. J Pediatr 2015;167(02):238-245
9 Graz B, Dicko M, Willcox ML, et al. Sublingual sugar for hypoglycaemia in children with severe malaria: a pilot clinical study. Malar J 2008;7:242

10 Bourchier D, Weston P, Heron P. Hypostop for neonatal hypoglycaemia. N Z Med J 1992;105(926):22

11 Brown LD, Rozance PJ. A sweet addition for the treatment of neonatal hypoglycemia. J Pediatr 2016;170:10-12

12 Bennett C, Fagan E, Chaharbakhshi E, Zamfirova I, Flicker J. Implementing a protocol using glucose gel to treat neonatal hypoglycemia. Nurs Womens Health 2016;20(01):64-74

13 Weston PJ, Harris DL, Battin M, Brown J, Hegarty JE, Harding JE. Oral dextrose gel for the treatment of hypoglycaemia in newborn infants. Cochrane Database Syst Rev 2016;(05):CD011027

14 WHO. The World Health Organization's infant feeding recommendation; January 2015. Available at: http://www.who.int/ nutrition/topics/infantfeeding_recommendation/en/. Accessed November 8, 2017

15 Rozance PJ. Update on neonatal hypoglycemia. Curr Opin Endocrinol Diabetes Obes 2014;21(01):45-50

16 Boluyt N, van Kempen A, Offringa M. Neurodevelopment after neonatal hypoglycemia: a systematic review and design of an optimal future study. Pediatrics 2006;117(06):2231-2243

17 Harris DL, Weston PJ, Harding JE. Incidence of neonatal hypoglycemia in babies identified as at risk. J Pediatr 2012;161(05):787-791

18 Special Care Nursery Admissions. Available at: https://www. marchofdimes.org/peristats/pdfdocs/nicu_summary_final.pdf. Accessed June 29, 2017

19 Stanley CA, Baker L. The causes of neonatal hypoglycemia. N Engl J Med 1999;340(15):1200-1201

20 McKinlay CJD, Alsweiler JM, Ansell JM, et al; CHYLD Study Group. Neonatal glycemia and neurodevelopmental outcomes at 2 years. N Engl J Med 2015;373(16):1507-1518

21 Harris DL, Alsweiler JM, Ansell JM, et al; Children with Hypoglycaemia and their Later Development (CHYLD) Study Team. Outcome at 2 years after dextrose gel treatment for neonatal hypoglycemia: follow-up of a randomized trial. J Pediatr 2016;170:54-59

22 Screening guidelines for newborns at risk for low blood glucose. Paediatr Child Health 2004;9(10):723-740

23 Rawat M, Chandrasekharan P, Turkovich S, et al. Oral dextrose gel reduces the need for intravenous dextrose therapy in neonatal hypoglycemia. Biomed Hub 2016;1(03):448511

24 Breast Feeding Report Card United States 2014. Available at: https://www.cdc.gov/breastfeeding/pdf/2014breastfeedingreportcard.pdf. Accessed July 1, 2017

25 Harris DL, Gamble GD, Weston PJ, Harding JE. What happens to blood glucose concentrations after oral treatment for neonatal hypoglycemia? J Pediatr 2017;190(July):136-141 